\title{
The Riddle of the Red Rectangle
}

\author{
Vincent Icke \\ Sterrewacht Leiden, Postbus 9513, 2300 RA Leiden, The Netherlands
}

\begin{abstract}
I reconsider my study (Icke 1981) of the hydrodynamic mechanism that is responsible for the shape of the Red Rectangle. The density of this nebula is so high, and the central star(s) so cool, that momentum driven outflow is almost guaranteed. Focusing of a spherically symmetric stellar wind through an inner shock creates a biconical pattern that may be responsible for the characteristic X-shape of the outer nebula. I compute the evolution of periodic biconical outflow. Observations made available to me by Van Winckel can thus be modeled in great detail. Features that I initially thought to be problematic for my interpretation, such as the occurrence of intersecting shocks on the symmetry axis, turned out to be present on closer inspection of the $\mathrm{H} \alpha$ image.
\end{abstract}

\section{Bipolar nebulae}

In previous investigations of bipolar nebulae, I have concentrated on disk-wind interactions that are 'energy driven'. A central star blows a spherical wind into a toroidal nebula; the wind has such a low density and high speed that the surrounding (inner) shock generates a very hot, pressure dominated layer that then drives the outer shock. Because flow in the high pressure region is very subsonic, inhomogeneities in this layer are mostly smoothed out, so that the outer shock and the nebular shape are well rounded.

This flow pattern, obtained if the Poisson exponent for adiabatic flow is $\gamma=5 / 3$, can easily explain the appearance and behaviour of a large class of bipolar nebulae (Balick 1987; Balick, Icke \& Preston 1987). In fact, the shape $r(\theta, t)$ of the outer shock can be found analytically from the equation

$$
\frac{\partial r}{\partial t}=\left\{\frac{(\gamma+1) P}{2 \rho}\left[1+\left(\frac{1}{r} \frac{\partial r}{\partial \theta}\right)^{2}\right]\right\}^{1 / 2}
$$

(Icke 1988). Here $P$ is the pressure behind and $\rho$ the gas density ahead of the shock. If the density distribution can be written as a product of a function $K(\theta)$ of the polar angle $\theta$ and the inverse square of the radial distance $r$, which is plausible for constant-velocity stellar winds, the solution of this equation is

$$
\rho(r, \theta)=r^{-2} K(\theta) ; \quad \log r=E t-\int \sqrt{E^{2} \cdot K(\theta)-1} d \theta+g(E)
$$

with separation constant $E$. This form allows the easy generation of predicted shock shapes for a great variety of confining density distributions. 

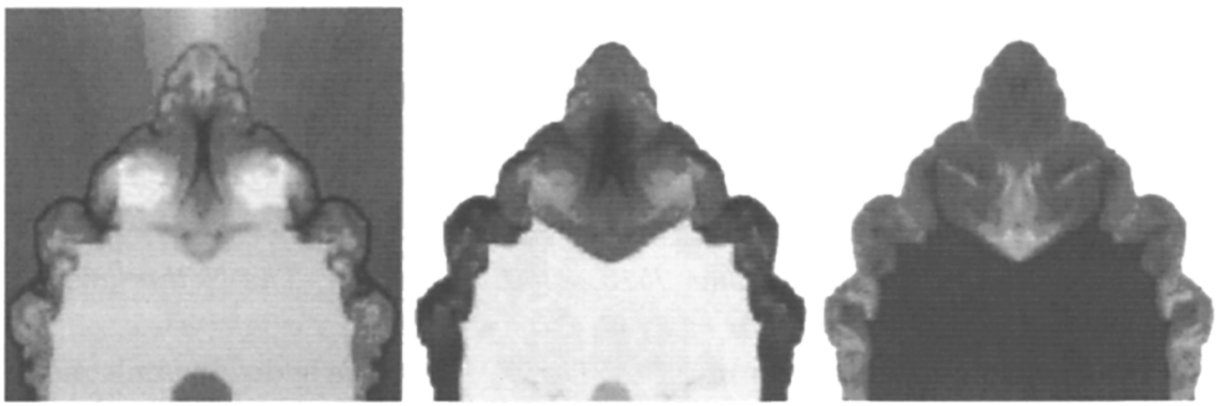

Figure 1. Typical bipolar model in the case $\gamma=1.1$. From left to right, the grayscale indicates the gas density, pressure, and absolute velocity. The symmetry axis runs vertically, the disk plane is at the bottom. Notice the instabilities that develop in the momentum driven layer, and the formation of a 'FLIER' in the polar cap.

If the flow is strongly compressible, a thick high-pressure smoothing shell does not develop. This regime is often called 'momentum driven'. In such flows the gas still has considerable momentum after passage through the inner shock, and thereby shapes the outer nebula by direct impact rather than by a mediating pressure. Strong cooling can provide the requisite compressibility, and instabilities will certainly occur. In order to allow dimensionless scaling of the simulations, I have for the moment opted to mimic the high compressibility by picking a small value of the Poisson constant (usually $\gamma=1.1$ ). A typical result is shown in Fig.1. All computations reported here were obtained with my FCT/LCD hydrocode (Icke 1991) on a rectangular-cylindrical mesh.

\section{Shape of the inner shock}

In my work on the shaping of bipolar nebulae I noted that the inner (reverse) shock surrounding disk-constrained outflow sources can take a large variety of shapes, and that this might lead to focusing effects (Icke 1988). Remarkable deviations from simple convex shapes can occur, due to the following instability, which is most clearly seen in movies of the evolving flow. First, the shock takes the form of a prolate ellipsoid, due to the obstruction of the disk. The oblique shock conditions produce a faster postshock speed at intermediate latitudes. This creates an incipient biconical flow. These faster postshock streams produce inward curving shocks that intersect on the symmetry axis. This, in turn, creates a high pressure pocket that dints the inner shock at the poles. This deforms the shock even more, eventually producing a series of secondary shocks that collide on the axis and make the pressure pocket almost stagnant. Around this self-created obstruction, the biconical flow streams outward (Fig. 2). 


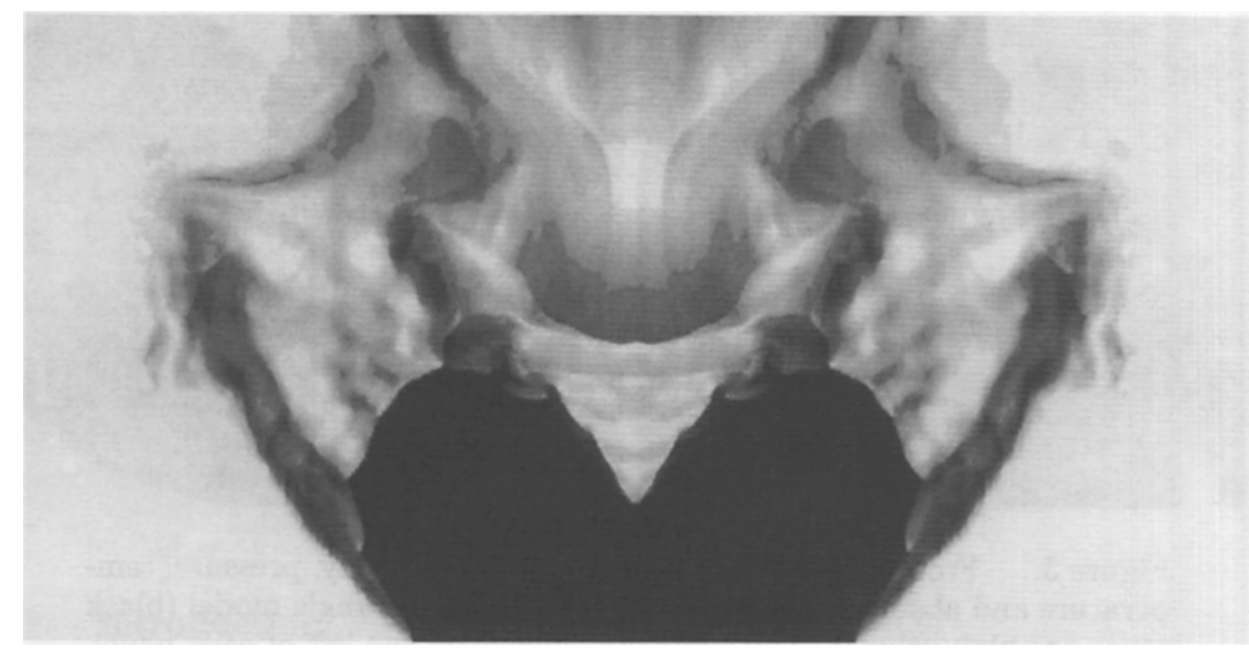

Figure 2. Velocity field around a spherically symmetric outflow that impinges on a thin hyperboloidal disk. Symmetry axis is the central vertical, the equatorial plane is at the bottom. Grayscale indicates the absolute value of the flow velocity. The dark intermittent plumes that extend beyond the strongly aspherical shock front form a cone that remains supersonic, due to the Hugoniot jump across an extremely oblique shock. The triangular structure pointing downward is a stagnation pocket caused by shocks colliding on the axis.

\section{Biconical flow}

In previous work, I studied the possibility that biconical flow is driven by a ring of high surface brightness in an accretion disk (Icke 1981). While this mechanism might work in some sources, new observations (Van Winckel 2002, in preparation) rule it out in the case of the Red Rectangle. In these images, it is clearly seen that the RR flow is intermittent, which is difficult to produce in a steady accretion disk. Accordingly, I tried to see what happens as a consequence of focusing by the inner shock. In many simulations this inner shape is seen to vary in a quasi-regular way, thereby modulating the biconical outflow. Because my current hydro methods do not allow me to resolve the inner and the outer regions simultaneously, I assumed periodic biconical inflow as an inner boundary condition, and studied its consequences for the evolution of the outer nebula.

\section{The Red Rectangle}

The density of the Red Rectangle is so high, and the central star(s) so cool, that radiative losses will be considerable, and highly compressible (momentum driven) outflow is almost guaranteed. Focusing of the primary gas through the inner shock may create a biconical outflow pattern that can produce the characteristic X-shape of the outer nebula. 

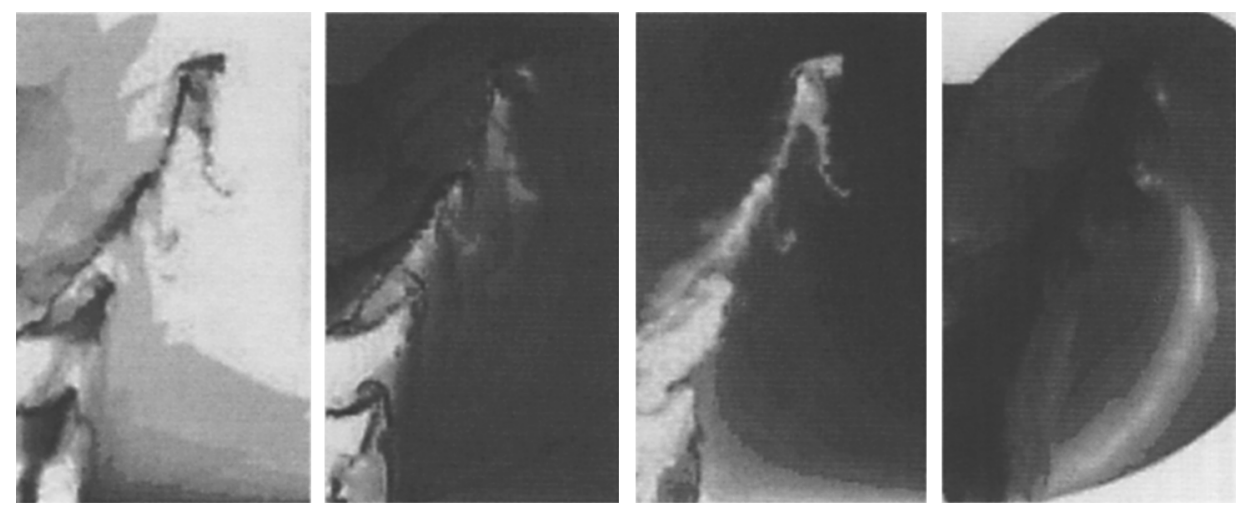

Figure 3. From left to right: logarithm of the density, pressure, temperature and absolute velocity of a typical Red Rectangle model (black tones are high values). The symmetry axis is on the left of each panel, the disk plane is at the bottom. Grid size is $300 \times 500$. Poisson index $\gamma=1.1$. Disk: initial density 5, pressure 1. Conical outflow: opening angle $25^{\circ}$, density 30 , pressure 1 , Mach number 35 , duration on-state 0.02 , off-state 0.02. Atmosphere: density 1, pressure 1 (dimensionless units).

Observations made available to me by Van Winckel can thus be modeled in great detail. Computational experiments show that a specific set of features is common to a wide range of compressible biconical flows. Key features are (Fig.3): (1) cup-shaped density ridges close to the central source, which become ragged rings at higher altitude; (2) the recurving bow shocks ahead of the gaseous rings that are shot upwards from the centre; (3) intersecting shocks on the symmetry axis, forming Mach stems ('X-shocks'); (4) the gradual widening of the effective cone opening angle at higher altitudes, due to the outward pressure behind these shocks. One feature that I initially thought to be problematic for my interpretation, namely the occurrence of $\mathrm{X}$-shocks on the axis, turned out to be present on closer inspection of an $\mathrm{H} \alpha$ image sent by Van Winckel.

I have constructed a sequence of projections of the density and of velocity channel maps for comparison with observations. Full colour-coded versions of the flow patterns can be downloaded from www.strw.LeidenUniv.nl/ icke/. QuickTime movies of some of my simulations can be obtained on request from icke@strw.LeidenUniv.nl.

\section{References}

Balick, B. 1987 AJ 94, 671

Balick, B., Icke, V., \& Preston, H.L. 1987 AJ 94, 1641

Icke, V. 1981 ApJ 247, 152

Icke, V. 1988 A\&A 202, 177

Icke, V. 1991 A\&A 251, 369 